\title{
Impacto del número de controles periódicos en la salud bucal de los pacientes atendidos en la Clínica de Odontopediatría
}

\author{
Impact of periodic controls number on the oral health of \\ patients treated at the Pediatric Dentistry Clinic \\ Impacto do número de controles periódicos na saúde bucal dos \\ pacientes atendidos na Clínica de Odontologia Pediátrica
}

\author{
Silvia Sosa Torices ${ }^{1}$ (DD 0000-0003-2581-488X \\ Ramón Ávarez-Vaz' (D) 0000-0002-2505-4238 \\ Fernando Massa² (D) 0000-0002-2922-4047 \\ María del Carmen López Jordi3 ${ }^{3}$ (D) 0000-0002-9025-3188 \\ Judith Liberman ${ }^{4}$ iD 0000-0002-6560-9146
}

\section{Resumen}

La Clínica de Odontopediatría desarrolla un modelo de atención con énfasis en promoción, educación y rehabilitación destacándose su control y mantenimiento. No hay información sobre el impacto de los controles periódicos.

Objetivo: Evaluar la asociación del número de controles y la salud bucal de niños entre 5 y 10 años. Estudio transversal, descriptivo (2017-18) y retrospectivo (hasta 2014) en dos subpoblaciones: $\mathrm{G} 1=$ controles y $\mathrm{G} 2$ =primera vez, evaluando diferencias de piezas afectadas

Resultados: 115 niños, 44 en G1 y 71 en G2. El 100\% presentaron biopelícula. G1 presentó un valor significativamente menor del IPV $>20 \%(p<0.001)$, de lesiones cavitadas $(p<0.001)$. G1 con 2 o más controles el promedio de lesiones iniciales fue de 2,6 y G2 de 4,5 ( $\mathrm{p}<0.001$ ). Conclusiones: Los niños con dos o más controles presentaron mejor situación de salud bucal que quienes consultaron por primera vez. Se confirma la importancia del control programado para el mantenimiento de la salud bucal.

Palabras clave: Salud oral, modelo de atención, revaluación de la salud, controles odontológicos, chequeos dentales.

Cátedra de Odontopediatría, Facultad de Odontología, Universidad de la República, Uruguay. drasilviasosatorices@gmail.com

Servicio de Epidemiología y Estadística. Facultad de Odontología, Universidad de la República. Uruguay.

Facultad de Odontología, Universidad de la República, Uruguay.

Cátedra de Odontopediatría, Facultad de Odontología, Universidad de la República, Uruguay.

Fecha de recibido: xxxxxxxxxxx - Fecha de aceptado: $x x x x x x x x x x$ 


\section{Abstract}

The Pediatric Dentistry Department develops a model of care with emphasis on promotion, health education and rehabilitation highlighting their control and maintenance. There is no information about the impact of regular checks.

Objective: To evaluate the association of the number of annual controls and oral health of children between 5 and 10 years. Transversal and descriptive study (2017-18) and retrospective (until 2014) in two subpopulations: G1-controls and G2-first time, evaluating differences in affected teeth.

Results: 115 children, 44 G1 and 71 G2. $100 \%$ of children had biofilm. G1 had a significantly lower IPV value $>20 \%(\mathrm{p}<0.001)$ and cavitated lesions $(\mathrm{p}<0.001)$ than G2. In $\mathrm{G} 1$ with 2 or more controls the average of initial lesions was 2.6 and 4.5 in G2 $(\mathrm{p}<0.001)$.

Conclusions: Children with two or more controls had a better oral situation that those who attend for the first time. This confirms the importance of scheduled control for oral health maintenance.

Keywords: Oral health, model attention, health reassessment, dental controls, dental checkups.

\section{Introducción}

La Clínica de Odontopediatría de la Facultad de Odontología (FO), Universidad de la República (Udelar), desarrolla un modelo de atención con énfasis en la promoción, la importancia de la educación para la salud del niño y/o adolescente y su grupo familiar, el control de las enfermedades bucales más prevalentes y procedimientos rehabilitadores concebidos dentro

\section{Resumo}

A Clínica de Odontologia Pediátrica desenvolve um modelo de cuidado com ênfase na promoção, educação em saúde e reabilitação destacando seu controle e manutençấo. Não há informações que sustentem o impacto que os controles regulares.

Objetivo: Avaliar a associação do número de controles anuais e da saúde bucal de crianças entre 5 e 10 anos. Estudo transversal e descritivo (2017-18) e retrospectiva (até 2014) em duas subpopulações: G1-controle e G2primeira vez.

Resultados: 115 crianzas: G1-44 e G2-71. $100 \%$ do de crianças apresentaram biofilme. G1 apresentou valor de IPV $>20 \%$ e lesóes cavitadas significativamente menor $(p<0,001)$. G1 com 2 ou mais controles a média de lesões iniciais foi de 2,6 e no G2 $4,5(\mathrm{p}<0,001)$.

Conclusóes: Crianças que assistem a 2 ou mais controles têm uma melhor situaçáo de saúde bucal em comparação com aquelas que consultam pela primeira vez. Confirmase a importância do controle programado para manutençáo da saúde bucal.

Palavras-chave: Saúde bucal, modelo de atenção, reavaliação da saúde, controles dentários, exames odontológicos.

de una filosofía preventiva en la cual se destaca el control y mantenimiento. El presente estudio aborda un análisis en profundidad, actual y retrospectivo de pacientes entre 5 y 10 ańos que asistieron a la Clínica de Odontopediatría, durante los ańos 2017 y parte del 2018 y la relación entre el cumplimiento del examen de revaluación o recitación y la salud bucal de los mismos. Siguiendo a Bordoni y col. ${ }^{(1)}$ el examen de revaluación de la situación de salud bucal tiene 
como principal componente el seguimiento de las enfermedades producidas por placa microbiana o biopelícula que son altamente dependientes del comportamiento personal. Deep P. (2) plantea que el examen de revaluación provee un régimen de cuidados continuados dirigidos a la preservación de la salud bucal y la planificación del tratamiento futuro, delimitando la progresión y los efectos de las enfermedades bucales lo más precozmente posible. Wange y Holts ${ }^{(3)}$ informan sobre la necesidad de realizar un monitoreo regular de las lesiones iniciales y el acompańamiento de los estados de desarrollo dentario en niños para garantizar que las intervenciones sean adecuadas y oportunas y para la detección precoz de las manifestaciones bucales de enfermedades sistémicas, entre otros. Las instancias de revaluación prevén el asesoramiento, la motivación y el refuerzo de las orientaciones preventivas de contribuir al mantenimiento de la actitud positiva hacia la salud del paciente. Dos estudios expresan que el período ideal para el examen de revaluación plantea diferencias entre países y sistemas de salud, aunque un intervalo de 6 meses ha sido aceptado como lapso ideal ${ }^{(4-5)}$. Cada niño posee condiciones clínicas y necesidades de tratamientos diferentes generando la exigencia de que el profesional planifique estrategias de control, cuidados preventivos y de tratamientos diferenciados en base a la evaluación de riesgo y actividad de cada paciente para ofrecer regímenes de prevención y tratamiento eficaces y al mismo tiempo evitar el sub o sobre tratamiento. A pesar de ello, no existe evidencia científica conclusiva sobre el intervalo confiable para la recitación y el beneficio de la práctica de revaluar todos los pacientes cada 6 meses ha sido cuestionado. Mettes ${ }^{(6)}$ realizó un estudio en Cochrane Library y concluyó que no hay evidencia suficiente para asegurar sobre el potencial benéfico del intervalo de los controles odontológicos. En el Reino Unido, el National Institute for Clinical Excellence (NICE) ${ }^{(7)}$, estableció en el 2004 que un intervalo menor a tres meses y uno mayor a 12 meses para la re- citación resultan inadecuados. La mencionada guía toma en cuenta el bienestar de las personas, la salud general y los hábitos preventivos, la incidencia de caries y la salud periodontal. La meta que plantea es mejorar el mantenimiento de la calidad de vida de los pacientes y reducir la morbilidad asociada con las enfermedades bucales. Las recomendaciones del NICE para los intervalos de revaluación de la situación oral son los siguientes:

- Para todos los pacientes el intervalo más corto debe marcarse en tres meses (la recitación antes de los tres meses no es una necesidad que deba indicarse por rutina, en niños, puede ser necesario en algún caso particular, situación de urgencia, episodios que requieren un cuidado especial).

- Para menores de 18 años el intervalo más largo de recitación debe ser de doce meses (hay evidencia que la progresión de la caries dental puede ser más rápida en niños y adolescentes que en adultos y el desarrollo de la dentición debe ser también evaluado).

- El período entre los controles en mayores de 18 años puede extenderse a los 24 meses. Intervalos más largos no son recomendables ya que actúa en contra de la relación odontólogo-paciente y el estilo de vida de la persona puede cambiar en un período tan prolongado.

- El profesional debe discutir la recomendación que establece para el intervalo del control con el paciente explicándole los motivos y si es posible modificarlo en el futuro.

Se recomienda que la indicación del intervalo adecuado debe ser analizado para cada paciente según riesgo y actividad. ${ }^{(8)} \mathrm{La}$ importancia del control periódico en la atención de la salud conlleva a que los servicios de salud públicos y privados lo implementen como rutina de tratamiento ya que permite ofrecer regímenes de prevención adecuados de acuerdo con la situación de salud revaluada y terapias más eficaces a riego mínimo de sub o sobre tratamiento. 


\section{Objetivos}

Objetivo general. Evaluar la asociación del número de controles anuales odontológicos con la salud bucal de los pacientes atendidos en la Clínica de Odontopediatría, FO, Udelar.

Objetivos específicos. -Cuantificar la presencia o ausencia de placa microbiana, caries dental y gingivitis en la población de niños que concurre a los controles periódicos establecidos y en la población que demandan asistencia por primera vez. -Evaluar el gradiente de enfermedad de las variables dependientes (biopelícula, caries dental e inflamación gingival) e independientes (motivo de consulta, sexo, edad, número de controles, cobertura de salud, frecuencia de cepillado), con los controles periódicos.

\section{Metodología}

Se realizó un estudio de diseño transversal, descriptivo y analítico sobre el análisis actual del total de pacientes entre 5 y 10 ańos, asistidos y evaluados durante el período 2017-2018 y el análisis retrospectivo hasta el 2014, en la Clínica de Odontopediatría, FO, Udelar.

Recolección de datos. Realizada entre mayo y octubre de 2017 y febrero de 2018, incluyó además el análisis retrospectivo de las historias clínicas de los pacientes asistidos entre 2014 y 2017, la aplicación de un cuestionario, y un examen clínico, descritos a continuación. Historias clínicas: se relevaron los siguientes datos: fecha de nacimiento, sexo, año de inicio de la atención en la clínica de Odontopediatría, y cantidad de controles periódicos anuales recibidos. Cuestionario: de tipo estructurado a los padres o responsables de los niños seleccionados, conteniendo preguntas sobre características socioeconómicas y la cobertura de salud. Examen clínico: fue realizado por un solo operador previamente entrenado y calibrado, (Kappa y reproductibilidad intraexaminador $=0.72$ ) en la unidad dental de la clínica de Odontope- diatría con el uso de espejo plano sin aumento y sonda milimetrada CPI de la OMS.

Población de estudio. Se incluyeron los pacientes entre 5 y 10 años sin padecimientos sistémicos que concurrieron a control odontológico (G1) y aquellos que demandaron asistencia por primera vez (G2) en la Clínica de Odontopediatría entre abril de 2017 y febrero de 2018. Fueron excluidos pacientes con padecimientos sistémicos y cuyos padres/responsables no firmaron el consentimiento informado.

Variables de estudio en relación con la salud oral:

\section{Variables dependientes}

A) Biopelícula evaluado a través del Índice de Placa Visible de Löe \& Silness simplificado (IPV) ${ }^{(9)}$, siendo código $0=$ ausencia de biofilm (placa) visible; $1=$ presencia de biopelícula (placa) visible y para establecer el acúmulo de biopelícula no compatible con salud usamos el IPV $>20 \%$.

B) Prevalencia y extensión de caries dental determinado a través de los índices CPO e ICDAS $(10,11)$ siendo clasificadas las superficies en códigos: 0- superficie sana; 1 - lesión no cavitada activa; 2- lesión no cavitada inactiva; 3- microcavidad en esmalte; 4- sombra; 5- cavidad en dentina; 6- destrucción coronaria; 7-perdido por caries; 8- perdido por traumatismo; 9- ausente; 10- restauración adecuada; 11 - restauración alterada; 12 - restauración con lesión adyacente o sustitución indicada. Dentro de los acuerdos operacionales a los efectos del análisis estadístico de la prevalencia de caries dental se acuerda que $\mathrm{CPO}+3$ = valores de ICDAS 3, 5 , 6 (lesiones moderadas).

C) Inflamación gingival determinado a través del Índice de Sangrado Gingival (ISG) ${ }^{(12)}$ siendo los códigos: 0 - ausencia de sangrado marginal al sondaje; 1 - presencia de sangrado marginal al sondaje 


\section{Variables independientes}

A) Motivo de consulta: Código 0- solicita asistencia por primera vez en la clínica; Código 1citado para control: Código 2- demanda asistencia en situación de urgencia.

B) Sexo: 0- femenino; 1- masculino.

C) Número de controles. Códigos: $1=$ una consulta de control; $2=$ dos o más consultas de control

D) Cobertura de salud: Códigos 0-2 Sector Público: (0- ASSE; 1- Sanidad Militar; 2-Sanidad Policial); y 3-4 Sector Privado: (3-FONASA y 4- Seguros privados)

E) Frecuencia de cepillado bucal: Código 1- Insuficiente (< dos cepillados/día); 2- Suficiente ( $\geq$ dos cepillados/día)

\section{Consideraciones éticas}

El proyecto de investigación fue aprobado por el Comité de Ética de la FO, Udelar, expediente 251/16. El equipo investigador se comprometió a mantener la confidencialidad de los datos obtenidos. Los responsables de los niños fueron informados sobre los objetivos del estudio y se les solicitó la firma del consentimiento libre informado, el asentimiento de los niños antes de ser examinados (no firma) y el manejo de historias clínicas, cumpliendo con la ley 18335/008 y con el Decreto 379/008 y la ordenanza 2010.

\section{Análisis estadístico}

Los datos recogidos fueron analizados a través del software R Core Team 2019 La descripción de las variables de índole cuantitativa se llevó a cabo mediante promedios mientras que se calcularon proporciones en los casos concernientes a variables binarias. Se confeccionaron gráficos de caja para comparar la distribución de las variables cuantitativas entre los grupos conformados por las variables cualitativas. La asociación entre la situación de salud oral y el número de controles fue evaluada en ambas subpoblaciones, a través de la prueba no paramétrica de Wilcoxon para grupos independientes (en el caso de variables cuantitativas). En el caso de variables binarias se empleó las pruebas exactas de Fisher para variables binarias y se calcularon Odds-Ratio (OR) ${ }^{(13)}$. Se determinó significación estadística a partir de un nivel de 5\%.

Hipótesis. Existe diferencia en la situación de salud bucal entre los niños que concurren a control periódico y los que solicitan asistencia por primera vez.

\section{Resultados}

Un total de 115 niños participaron, siendo 44 pertenecientes al G1 y 71 al G2. La distribución del total de niños por año de nacimiento resultó equilibrada: 60 niños nacidos entre 2007-2009 y 55 entre 2010-2012. En el total de la muestra resultaron 54 mujeres y 61 varones. La integración en los dos grupos mostró una distribución por sexo balanceada: 22 niñas y 22 varones en el G1 (50\% cada uno) y 32 niñas (45\%) y 39 (55\%) varones en el G2. La distribución del número de consultas de control registradas en las historias clínicas mostró que $93.2 \%$ de los niños poseen 1,2 o 3 controles y el restante $6.8 \%, 4$ consultas. La distribución del número de consultas de control en el G1 arrojó que el $36.4 \%$ presentó 1 consulta de control, el $34.1 \%$ dos, el $22.7 \%$ tres y el $6.8 \%$ restante cuatro En lo referente a la cobertura de salud, $35.7 \%$ poseía cobertura de asistencia privada y $64.3 \%$ cobertura en el sistema público.

\section{Distribución de las distintas variables en ambos grupos}

A) Biopelícula. El IPV acumulado fue muy superior en el grupo de nińos que consultaban por primera vez comparado a los que concurren a control periódicamente $(66 \%-18 \%, \mathrm{p}<0,001)$. Cuando analizamos el acúmulo de biopelícula no compatible con salud (IPV>20\%) los niños acumulan más biopelícula que las niñas, aunque la diferencia no es significativa (54\% $41 \%, \mathrm{p}=0.191$ ). El promedio de placa visible de los niños con cobertura de asistencia en el sector público fue significativamente mayor que los de 
asistencia en el sector privado $(14.1 \%-12.1 \%$, $\mathrm{p}=0.007)$. (Gráfico 1).

\section{Gráfico 1: Distribución de biopelícula según tipo} de cobertura de salud

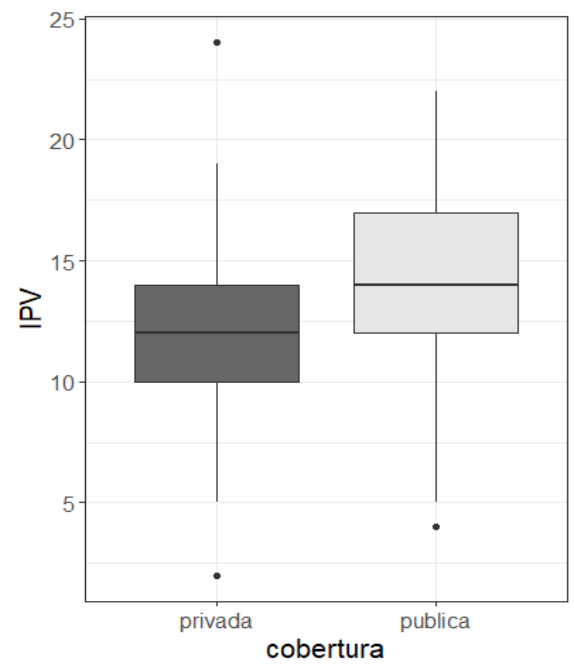

B) Inflamación gingival. Se analizó la prevalencia de sangrado gingival según el motivo de consulta, observándose un valor ligeramente superior en el grupo G2 (Tabla 1). En cuanto al sexo, la prevalencia de sangrado gingival fue mayor en el grupo de niñas (Tabla 2).

Tabla 1: Prevalencia de sangradogingival según tipo de participante

\begin{tabular}{|c|c|c|c|}
\hline & G1 & G2 & p-valor \\
\hline ISG & $11.3 \%$ & $12.7 \%$ & 0.998 \\
\hline
\end{tabular}

Tabla 2: Prevalencia de sangrado gingival según sexo

\begin{tabular}{|c|c|c|c|}
\hline & Fem & Masc & p-valor \\
\hline ISG & $14.8 \%$ & $9.8 \%$ & 0.417 \\
\hline
\end{tabular}

C) Caries dental. El 99.1\% de los niños presentan al menos una lesión de caries, correspondiendo el $70.4 \%$ a lesiones cavitadas. Al indagar sobre la asociación entre caries actual y el motivo de consulta, resultó que el grupo G2 poseía mayor número de piezas dentarias con lesiones cariosas comparado con el G1 (p $<0.001$ ) (Gráfico 2). Al indagar sobre la asociación entre lesiones iniciales y número de consultas de control, el grupo que posee dos o más controles posee menor promedio de las mismas ( $\mathrm{p}=0.033)$, (Gráfico 3).

\section{Gráfico 2: Distribución de caries actual según} motivo de consulta

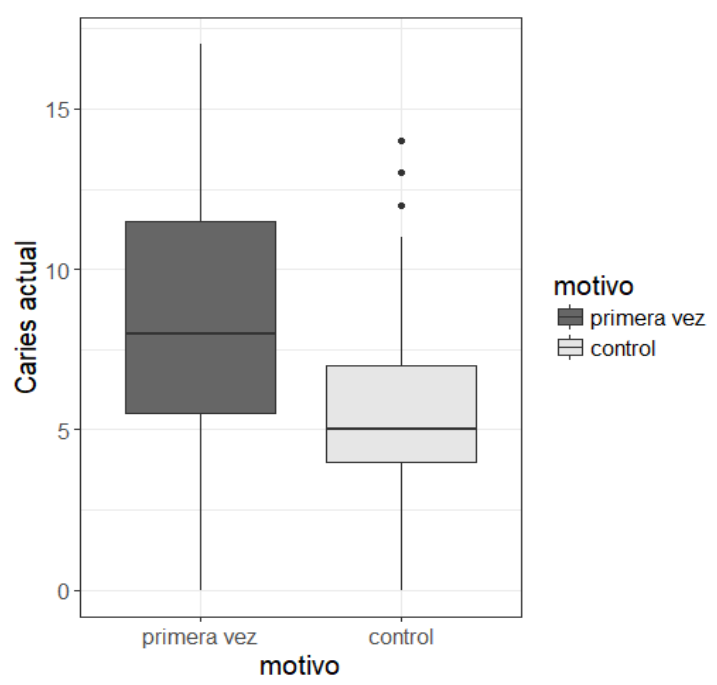

Gráfico 3: Distribución de lesiones iniciales según número de consultas de control

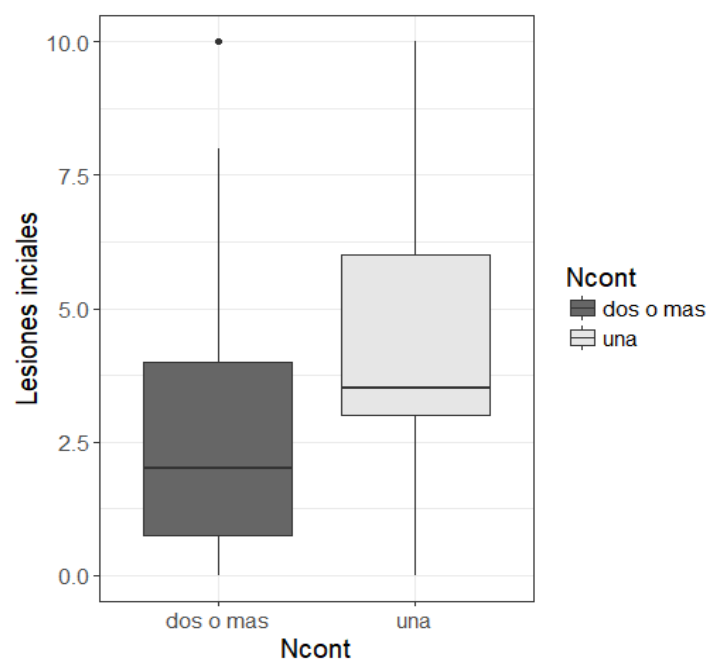


$\mathrm{Al}$ asociar las lesiones cavitadas en los grupos participantes según CPOD, resultó superior en G2 (4.8 - 1.1, p <0.001). El porcentaje de lesiones cariosas cavitadas en G1 fue inferior a G2 (38\% - 90\%, p<0.001), (Gráfico 4). Las lesiones cavitadas presentaron 14.52 veces más chance de progresar en el grupo G2 (OR: 5.4 39.02, IC 95\%). Un comportamiento similar se observó en la asociación entre las lesiones no tratadas con motivo de consulta, resultando un valor promedio muy superior en G2 (7.2-4.1, $\mathrm{p}<0.001$ ), (Gráfico 5). En los gráficos 6 y 7 se visualiza que dentro del G1, los nińos que tienen dos o más controles se comportan diferente en relación con las lesiones iniciales, pudiendo concluir que las lesiones no progresan en G1 y sí en G2 ya que hay más lesiones cavitadas. El promedio de piezas afectadas de caries fue de 6.3 para aquellos niños con cepillado insuficiente y 4.6 para los que tuvieron cepillado suficiente. La relación del cepillado con COPD+3 resultó en un valor promedio de 7.7 para los nińos con cepillado insuficiente y de 5.7 cuando el cepillado se consideró suficiente $(\mathrm{p}<0.025)$.

\section{Gráfico 4: Distribución de lesiones cavitadas} (CPO) según motivo de consulta

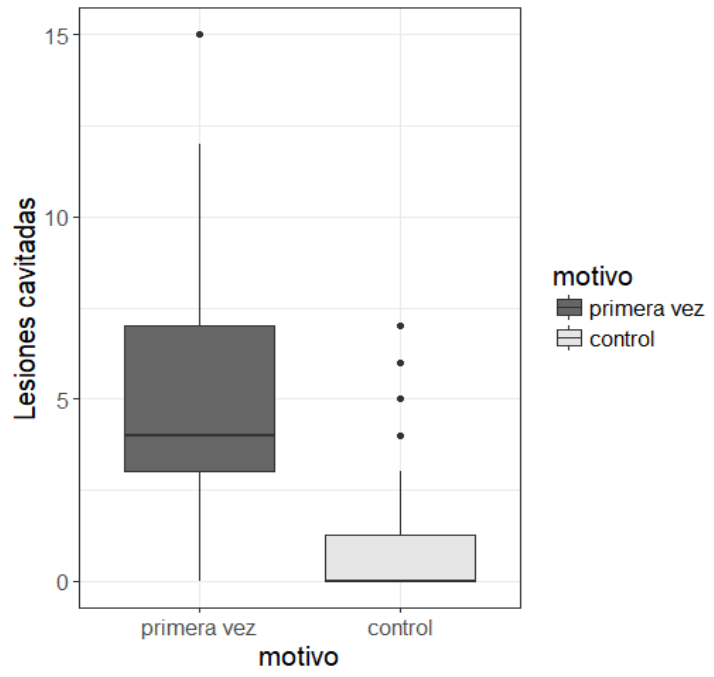

Gráfico 5: Distribución de lesiones cariosas no tratadas (CPO) según motivo de consulta

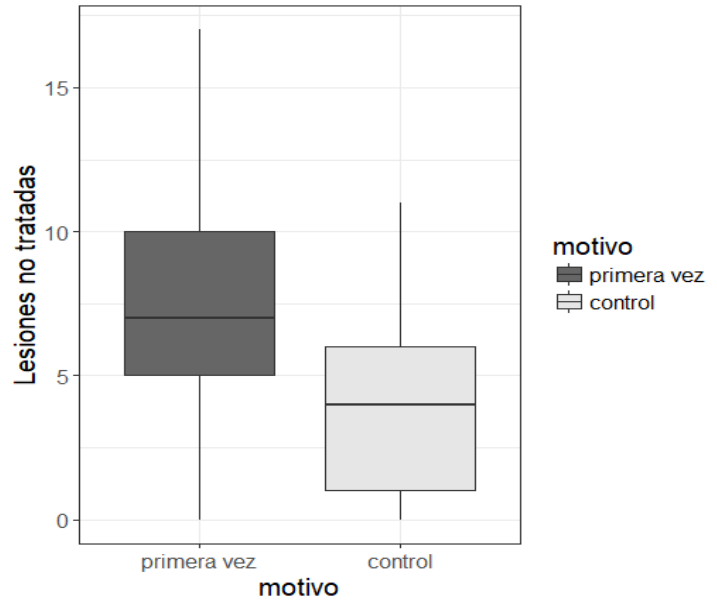

Gráfico 6: Distribución de CPO según cepillado

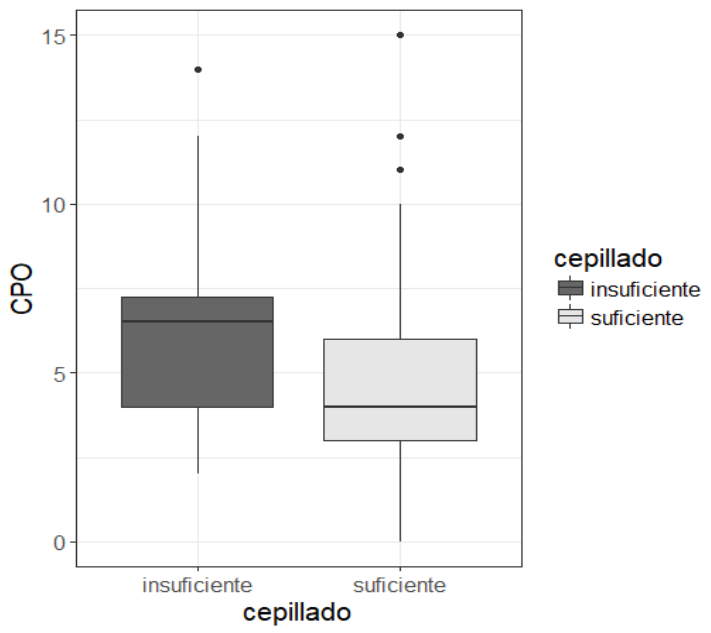

Gráfico 7: Distribución de CPO+3 según cepillado

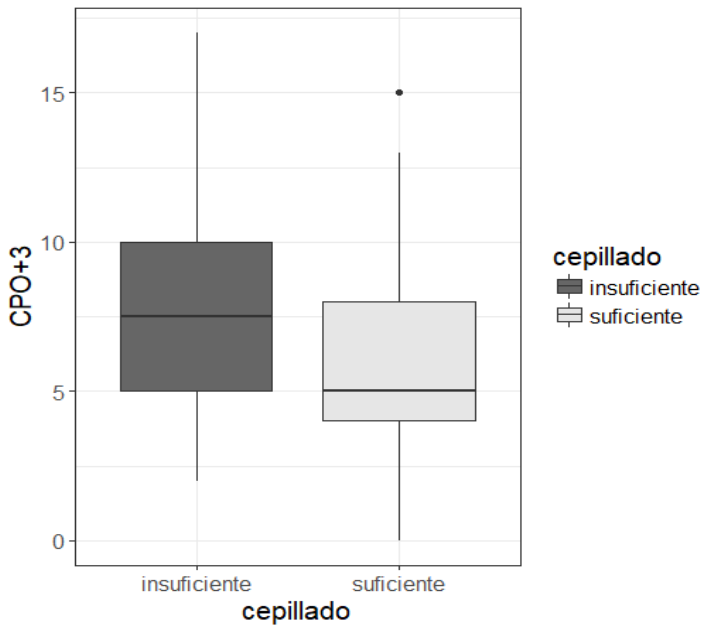




\section{Discusión}

Uno de los pilares fundamentales en Odontopediatría para el mantenimiento de la salud bucal es el retorno del paciente en un intervalo acordado junto al odontólogo tratante para evaluar su situación bucal. La American Academy of Pediatric Dentistry ${ }^{(14)}$ en el año 2013 establece que los intervalos de recitación deben ser asignados de acuerdo con las necesidades individuales de cada paciente teniendo en cuenta la incidencia de caries, el mantenimiento de restauraciones, la salud periodontal, hábitos preventivos, salud general e impacto en la calidad de vida de los individuos, evitando pérdidas dentarias, dolor y ansiedad. Siguiendo a Davenport y col ${ }^{(15)}$ se reconoce una compleja interacción de factores modificadores en el desarrollo y control de las enfermedades bucales que incluye: edad, nivel socioeconómico, uso del fluoruro, asistencia odontológica entre otros. Aunque la evidencia muestra que la higiene bucal realizada en forma mecánica es fundamental para la prevención y control de caries y enfermedad periodontal, es importante reconocer con Maltz y col. ${ }^{(16)}$ que el control óptimo del biofilm no es alcanzado por la mayoría de los individuos. En el presente estudio, que participaron 115 niños, siendo 44 pertenecientes al G1(control) y 71 al G2 (primera vez), todos los niños de ambos grupos presentaron biofilm, enfatizando la importancia que tienen las consultas periódicas para monitoreo y remotivación a fin de poder crear conductas saludables duraderas hacia la salud. Los procedimientos de higiene bucal son efectivos para controlar la enfermedad caries dental y asociada con el fluoruro representan dos componentes prioritarios en la atención de acuerdo con los criterios de mínima intervención ${ }^{(17)}$. Por su parte, en nuestro estudio el acúmulo de placa visible presentó un valor significativamente mayor en aquellos niños que concurren por primera vez. Esto estaría de acuerdo con Maltz ${ }^{(18)}$ que confirma que el monitoreo regular del control de placa es uno de los componentes que debe integrar el examen de control y recordatorio en Odontopediatría. La asociación de acúmulo de biofilm no compatible con salud con la cobertura de asistencia da valores significativamente mayores en los niños con cobertura del sector público. Si se considera que la cobertura del sistema de salud que tiene el niño representa de alguna manera el nivel socioeconómico de la familia, se podría generalizar que éste se asocia con una peor situación de salud bucal. De acuerdo con el National Institute of Health de Estados Unidos ${ }^{(19)}$, el bajo nivel socioeconómico se asocia con: un acceso limitado a los servicios, aspiraciones limitadas de salud bucal, baja auto eficacia y a la presencia de conductas de salud que aumentan el riesgo de caries. Se resalta entonces, la importancia del control periódico en la atención de la salud para el mantenimiento de la situación de salud bucal. Es poco consistente la evidencia en la literatura del efecto de los controles periódicos sobre el sangrado gingival, la gingivitis y aún la periodontitis. En el presente estudio, el diagnóstico de inflamación gingival se correlacionó con la presencia o ausencia de sangrado gingival ${ }^{(12)}$ y se observó un valor superior, aunque no significativo en los que concurren por primera vez. Es importante analizar que son variadas las causas que producen inflamación gingival en la población de nuestro estudio, tales como: una etapa muy dinámica de recambio dentario (movilidad, reabsorción) y una fase de erupción activa de múltiples órganos dentarios que de por sí generan una respuesta inflamatoria en los tejidos peridentarios. Es evidente que de sumarse la presencia de placa microbiana persistente y un cepillado insuficiente, los signos resultantes de inflamación son más notorios. Esto concuerda con lo establecido por Andrade y col. ${ }^{(20)}$ que al referirse a la etiología de la inflamación gingival en nińos y adolescentes acuerdan que "el acúmulo de placa microbiana, la erupción y la exfoliación dental, el recambio dentario y las influencias hormonales explican la inflamación gingival". La literatura científica plantea la asociación entre el efecto de los controles progra- 
mados y caries, pérdida de dientes y obturaciones en dentición caduca, mixta y permanente, aunque los resultados son poco consistentes ${ }^{(8,}$ 14, 15). En el presente estudio el porcentaje de niños con lesiones de caries cavitadas es inferior en los niños que concurren a controles periódicos y con una media de piezas cariadas también inferior comparada al grupo de los que consultan por primera vez. Estos resultados indican que los niños del grupo G2 están más enfermos y las lesiones podrían progresar libremente de no mediar tratamiento alguno. Por otro lado, al evaluar las lesiones iniciales de caries también se encuentran en mayor número en G2 que en G1 y al asociarlas con el número de controles se verifica que el niño que ha concurrido a dos o más controles tiene una mejor situación de salud bucal y que las lesiones iniciales no progresan. Los resultados obtenidos al relacionar las variables cepillado y caries dental concuerdan con el estudio de Tickle y col. ${ }^{(21)}$ que concluyeron que los niños de 5 años que visitaban en forma irregular al dentista tenían un mayor valor del índice ceod, distribuidos en más dientes perdidos y cariados y menos obturados. Reconocen que la asistencia regular al odontólogo ejerce un significativo efecto en el ceod, por lo que, los niños integrados a un programa preventivo de salud logran un efecto significativo en el mantenimiento de la salud bucal. De acuerdo con la concepción actual ${ }^{(22-23)}$, se expresa que cuando se verifica un aporte adecuado de fluoruros, es improbable que un niño libre de caries desarrolle lesiones profundas en un período de seis meses después del examen de reevaluación. En acuerdo con nuestro estudio, Abanto y col. (24) evaluaron la efectividad de un programa preventivo-asistencial en 351 nińos de 1 a 12 años y establecieron que por cada control habría un $77 \%$ de reducción del riesgo de aparición de nuevas lesiones de caries $(94,8 \%$ de niños no presentaron nuevas lesiones) y también, un aumento significativo en la probabilidad de regresión de las lesiones de caries iniciales activas. Concuerdan con nuestro estudio que, niños que habían concurrido al odontólogo previamente tenían lesiones de caries menos activas que los nińos que nunca habían visitado el dentista. Esto apoya la concepción de que un programa preventivo-asistencial en odontopediatría, debe estar dirigido a dos propósitos fundamentales:

1) lograr que la población infantil permanezca libre de caries y

2) en aquella con patología presente, promover su detención y/o regresión de los procesos cariosos.

Siguiendo a Clarkson, Pitts et al. ${ }^{(25)}$ se requiere más investigación para: mejorar y apoyar la comunicación paciente-odontólogo a fin de establecer un intervalo de controles variable basado en el diagnóstico riesgo y comprender el papel de la recuperación con la toma de decisiones a corto largo y largo plazo, basada en el riesgo, a fin de hacer un seguimiento y mantenimiento de la salud de los individuos.

Finalmente, confirmando esta filosofía de atención odontológica la reciente 74 Asamblea de OMS llevado a cabo en mayo 2021, establece entre sus recomendaciones, la necesidad de "reorientar el enfoque curativo tradicional, hacia un enfoque de promoción y prevención con identificación de riesgos a tiempo, atención integral e inclusiva, teniendo en cuenta a todas las partes interesadas para contribuir a la mejora de la salud bucal de la población con un impacto positivo en la salud general" (26).

\section{Conclusiones}

El presente trabajo representa el primer estudio dirigido a la evaluación de la eficacia de un programa preventivo-asistencial en la Facultad de Odontología, Udelar, basado en una filosofía de mínima intervención con controles periódicos programados. En este estudio el grupo de nińos con dos o más controles tiene una mejor situación de salud bucal, pudiendo concluir que las lesiones no progresaron mientras que los que consultan por primera vez están más enfermos y progresando la enfermedad libremente. 
Los niños integrados a un programa preventivo de salud logran un efecto significativo en el mantenimiento de la Salud Bucal. Es de vital importancia acordar intervalos de revaluación de acuerdo con las diferentes situaciones, cada niño posee condiciones clínicas y necesidades de tratamientos diferentes por lo que el profesional debe planificar estrategias de retorno, en base a la evaluación de riesgo de cada paciente para ofrecer tratamientos preventivos, no operatorios y operatorios eficientes y al mismo tiempo evitar el sub o sobre tratamiento. El análisis evaluativo de programas de atención odontológica aporta datos relevantes para la toma de decisiones y para que los servicios de salud públicos y privados implementen el control periódico programado como rutina de tratamiento.

\section{Referencias}

1. Bordoni N, Escobar A, Castilla R. Capítulo 42. En: Odontología Pediátrica. La salud bucal del niño y del adolescente en el mundo actual. $1^{\circ}$ Ed. Buenos Aires: Médica Panamericana, 2010. 881p.

2. Deep P. Screening for Common Oral Diseases. J Can Dent Assoc. 2000; 66:298-9.

3. Wange NJ, Holts D. Individualizing recall intervals in child dental care. Community Dentistry and Oral Epidemiology. 1995; 23 (1): 1-7.

4. Frame PS, Sawai R, Bowen WH, Meyerowitz C. Preventive dentistry: practitioners' recommendations for low-risk patients compared with scientific evidence and practice guidelines. Am J of Prev Med. 2000; 18 (2): 159-62.

5. Scott G, Brodeur JM, Olivier M, Benigeri M. Parental factors associated with regular use of dental services by second-year secondary school students in Quebec. J Can Dent Assoc. 2002; 68 (10): 604-8.

6. Mettes D. Insufficient evidence to support or refute the need for 6-monthly dental check-ups. What is the optimal recall frequency between dental checks? Evid Based Dent. 2005; 6 (3): 62-3.

7. NICE Guidance. Dental Checks: intervals between oral health reviews. October 2004. Disponible en: https://www.nice.org.uk/guidance/cg19

8. Gibson C, Moosajee A. Selecting appropriate recall intervals for patients in general dental practice an audit project to categorize patients according to risk. Dental Update. 2008; 35 (3): 188-190, 193-194.

9. Bordoni N, Doño R, Miraschi C. Preconc. Organización Panamericana de la Salud 1992.

10. ICDAS Foundation. International Caries Detection and Assessment System. 2016. Disponible en: https:// icdas.org/downloads.

11. Ekstrand KR, Gimenez T, Ferreira FR, Mendes F, Braga M. The International Caries Detection and Assessment System- ICDAS. A systemic review. Caries Res. 2018; 52 (5): 406-419.

12. Ainamo J, Bay I. Problems and proposals for recording gingivitis and plaque. Int Dent J. 1975; 25 (4): 229-35.

13. The R Project for Statistical Computing (OR), A language and environment for statistical computing. R Foundation for Statistical Computing, Vienna, Austria. URL: https://www.R project.org/

14. American Academy of Pediatric Dentistry (AADP). Guideline on Periodicity of Examination, Preventive Dental Services, Anticipatory Guidance/Counseling, and Oral Treatment for Infants, Children, and Adolescents. 2013. Disponible en: http://www.aapd.org/media/Policies_Guidelines/G_Periodicity.pdf

15. Davenport C, Elley K, Salas C, Taylor-Weetman CL, Fry-Smith A, Bryan S, Taylor R. The clinic effectiveness and cost of routine dental checks. NIHR Health Technology Assessment program. health Technol Access 2003. Disponible en: http://www.ncbi.nlm.nih.gov/pubmedhealth/PMH0015130/

16. Maltz M, Jardim JJ, Alves LS. Health promotion and dental caries. Braz Oral Res. 2010; 24 (Suppl 1): $18-25$.

17. Walsh L J, Brostek A M. Minimum intervention dentistry principles and objectives. Aust Dent J. 2013; 58 (Suppl 1): 3-16 
18. Maltz M, Andaló Tenuta LM, Groisman S, Cury JA. Cariología: Conceitos Básicos, Diagnóstico e Tratamento nao Restaurador. Publisher: 1era ed. Porto Alegre: Artes Medicas; 2016 144p.

19. US. Dept of Health and Human Services Healthy People 2010: midcourse review. Disponible en: http://www.healthypeople.gov/data/midcourse

20. Andrade y col. Epidemiología de las Enfermedades Periodontales en el Uruguay. Pasado y presente. Odontoestomatología. 2017; 19(30): 14-28

21. Tickle M, Williams M, Jenner T, Blinkhorn A. The effects of socioeconomic status and dental attendance on dental caries' experience, and treatment patterns in 5- year-old children. Br Dent J. 1999; 186 (3): 135-7.

22. Mejàre I, Källest $1 \mathrm{C}$, Stenlund H. Incidence and progression of approximal caries from 11 to 22 years of age in Sweden: A prospective radiographic study. Caries Res. 1999; 33 (2): 93-100.

23. Lith A, Lindstrand C, Gröndahl HG. Caries development in a young population managed by a restrictive attitude to radiography and operative intervention: II. A study at the surface level. Dentomaxillofac Radiol. 2002; 31(4): 232-9.

24. Abanto J, Celiberti P, Minatel M, Alvarez E, Cordeschi, Haddad A E, Bonecker M. Effectiveness of preventive program based on caries risk assessment and recall intervals on incidence and regression of initial caries lesions in children. Int J Paediatr Dent. 2015; 25(4): 291-9.

25. Clarkson JE, Pitts NB, Goulao B, Boyers D, Ramsay CR, Floate R, et al. Risk-based, 6-monthly and 24-monthly dental check-ups for adults: the INTERVAL three-arm RCT. Health Technol Assess 2020;24(60).

26. WHO 2021. Seventy-fourth World Health Assembly. EB148/SR/8. Mayo 2021. Disponible en: https://apps.who.int/gb/ebwha/pdf_files/EB148/B148_8-en.pdf

\section{Nota contribución de los autores:}

1. Concepción y diseño del estudio

2. Adquisición de datos

3. Análisis de datos

4. Discusión de los resultados

5. Redacción del manuscrito

6. Aprobación de la versión final del manuscrito

SST ha contribuido en $1,2,3,4,5$ y 6 .

RA ha contribuido en: $1,3,4$ y 6 .

FM ha contribuido en: 1, 3, 4 y 6 .

MCLJ ha contribuido en: 5 y 6 .

SC ha contribuido en: $1,3,4,5$ y 6 .

\section{Nota de aceptación:}

Este artículo fue aprobado por la editora de la revista Mag. Dra. Vanesa Pereira-Prado. 